\title{
Efecto de la aplicación de campos eléctricos sobre las interacciones entre los iones cloruro y la matriz de cemento
}

\author{
M. CASTELLOTE, C. ANDRADE, C. ALONSO \\ Instituto de Ciencias de la Construcción "Eduardo Torroja" (IETCC) CSIC, Madrid, España.
}

\begin{abstract}
La determinación de coeficientes de difusión de los iones cloruro a través del hormigón precisa en muchas ocasiones de la aplicación de campos eléctricos con el fin de obtener resultados en plazos razonables de tiempo. Sin embargo, esta aceleración del transporte podría modificar las complejas interacciones que tienen lugar entre los iones cloruro y la matriz de cemento, haciendo que los coeficientes obtenidos mediante ensayos de migración no fueran representativos de condiciones de difusión natural. Este aspecto se aborda en el presente trabajo mediante la realización de ensayos de migración de cloruros en estado estacionario y no estacionario, así como de difusión natural de cloruros y oxígeno en estado estacionario. Como resultado, se ha podido establecer que cuando el transporte se produce por migración, hasta un campo aplicado de $10.5 \mathrm{~V} / \mathrm{cm}$, ésta tiene lugar a través de poros del mismo tamaño que por los que se lleva a cabo la difusión natural, produciéndose una interacción más débil del adsorbato con el sustrato que en difusión natural, lo que debe tenerse en cuenta a la hora de diseñar los ensayos de migración para la determinación de los coeficientes de difusión de cloruros en estado no estacionario.
\end{abstract}

Palabras clave: campos eléctricos, pasta de cemento, iones cloruro, oxígeno, interacciones, alteraciones

\section{Effect of the application of electrical fields on the interactions between the chloride ions and the cementitious matrix}

The application of electrical fields to determine the chloride diffusion coefficients through concrete has become a need in order to obtain the required results in reasonable periods of time. However, this acceleration could modify the complex interactions between the chloride ions and the cementitious matrix, being the coefficients obtained by migration tests not representative of those of natural conditions. This subject is undertaken in this paper by performing steady and non-steady state migration tests for chlorides and natural diffusion for oxygen and chloride ions. As a result it has been stated that when applying an electrical field (until $10.5 \mathrm{~V} / \mathrm{cm}$ ), the transport of chloride ions take place through the same kind of pores, but the interaction to combine chlorides is smaller than when driven by natural diffusion. This is a point to be taken into account when designing the experimental conditions for migration tests to determine the non steady state diffusion coefficient of chloride ions.

Key words: electrical fields, cement paste, chloride ions, oxygen, interactions, alterations

\section{INTRODUCCIÓN}

La determinación de los coeficientes de difusión de los iones cloruro a partir de ensayos de migración está cada día más extendida, siendo ya una práctica habitual. Sin embargo, a pesar de haberse establecido la viabilidad teórica [1], todavía quedan muchos puntos sin clarificar, siendo uno de los más importantes el que no existe constancia de que la aplicación de campos eléctricos al hormigón no modifique las complejas interacciones que tienen lugar entre los iones cloruro y la matriz de cemento, haciendo que los coeficientes obtenidos mediante ensayos de migración no sean representativos de los que se tendrían en condiciones de difusión natural $[2,3]$.

A este respecto cabe considerar, por una parte, que el hecho de que los $\mathrm{Cl}^{-}$sean partículas cargadas, hace que sufran interacciones con la doble capa eléctrica de las paredes de los poros [4-8]. y como consecuencia, su difusión no sea tan rápida como la de otras especies neutras de su mismo tamaño $\left(\mathrm{O}_{2}\right)$, las cuales pueden pasar por poros de menor tamaño que aquellas [9-10]. Así, si al aplicar un campo eléctrico, la fuerza del mismo es capaz de vencer las interacciones electrostáticas de los cloruros con las paredes de los poros, éstos difundirían también a través de poros de menor tamaño, con el consiguiente aumento en su coeficiente de difusión respecto del obtenido por difusión natural, siendo similar al de la molécula de oxígeno.

Por otra parte, la capacidad de combinar cloruros por parte de una matriz de base cemento depende de múltiples factores, entre los que se encuentran la composición química de la fase acuosa presente en los poros de la pasta endurecida, el tipo de cemento, el uso de adiciones, el catión asociado y la concentración del ión cloruro [11-15]. Hoy en día, la mayor parte de los investigadores que trabajan en este campo están de acuerdo en que la retención de cloruros por parte de la pasta de cemento tiene lugar como resultado de una combinación de diferentes mecanismos, cuantificándose el fenómeno mediante isotermas de adsorción. Así, de ser la isoterma de combinación obtenida por migración de distinto tipo a la que da lugar la penetración por difusión natural, la influencia sobre los coeficientes de difusión obtenidos podría llegar a ser apreciable.

Estos aspectos se abordan en el presente trabajo. Para ello, se han llevado a cabo ensayos comparativos en estado estacionario de transporte de iones cloruro y molécula de oxígeno por difusión natural y migración de cloruros en estado estacionario. Adicionalmente, a partir de los datos de migración de cloruros en estado no estacionario, se ha calculado la isoterma de combinación y se ha comparado con una isoterma típica de difusión natural [16].

\section{EXPERIMENTAL}

Se amasaron probetas de hormigón y de pasta con el mismo tipo de cemento: $\mathrm{I}-45 \mathrm{~A}$, con la siguiente composición química: $\mathrm{SiO}_{2}: 19.37$ $\%, \mathrm{Al}_{2} \mathrm{O}_{3:} 6.12 \%, \mathrm{Fe}_{2} \mathrm{O}_{3}: 3.13 \%$, CaO: $62.86 \%, \mathrm{MgO}: 1.78 \%, \mathrm{SO}_{3}: 3.23$ $\%$, Cal libre: $1.28 \%, \mathrm{Na}_{2} \mathrm{O}: 0.18 \%$ y $\mathrm{K}_{2} \mathrm{O}: 1 \%$. Las probetas de hormigón fueron cilíndricas de $75 \mathrm{~mm}$ de diámetro x $150 \mathrm{~mm}$. de altura, y la 
dosificación empleada fue: cemento: $380 \mathrm{Kg} / \mathrm{m}^{3}$, Arena: $771 \mathrm{Kg} / \mathrm{m}^{3}$, Gravilla: $1177 \mathrm{Kg} / \mathrm{m}^{3}$, Relación agua/cemento: 0.4. Las probetas de pasta se prepararon en dos dimensiones distintas: 50 y $75 \mathrm{~mm}$ de diámetro, con una relación agua/cemento de 0.4 .

Transcurridas 24 horas desde su amasado se procedió a desenmoldar las probetas y a sumergirlas, para su curado, en una balsa de agua potable, en la que permanecieron durante 28 días. Al término del curado y hasta ensayo las probetas se conservaron sumergidas en disolución acuosa saturada de $\mathrm{Ca}(\mathrm{OH})_{2}$.

Con las probetas de pasta se llevaron a cabo ensayos de difusión natural y migración de cloruros en estado estacionario, así como de difusión de oxígeno en estado estacionario, según los procedimientos descritos en [17], [1] y [9] respectivamente. Con las probetas de hormigón se llevaron a cabo ensayos de migración de cloruros en estado no estacionario según el procedimiento descrito en [18].

\section{RESULTADOS Y DISCUSIÓN}

Los resultados obtenidos en los ensayos de pasta de cemento se muestran en la figura 1, donde se han representado conjuntamente los parámetros característicos de los tres ensayos realizados. Así, se presenta la evolución de la cantidad de ión cloruro en el compartimento anódico para el ensayo de migración (con escala en el eje $\mathrm{Y}$ derecho de la figura), la cantidad de cloruros que han pasado al compartimento destino en los ensayos de difusión natural, y la carga total acumulada registrada a los distintos tiempos en que se llevó a cabo el consumo del oxígeno en el cátodo (con escala en el eje Y izquierdo de la figura).

A partir de estos datos, se han calculado los coeficientes de difusión de los iones $\mathrm{Cl}^{-}$y de la molécula $\mathrm{O}_{2}$ a través de las probetas de pasta, siendo los resultados obtenidos de $3.62 \times 10^{-8} \mathrm{~cm}^{2} / \mathrm{s}$ para los iones cloruro en ensayo de difusión natural, de $3.85 \times 10^{-8} \mathrm{~cm}^{2} / \mathrm{s}$ para los iones cloruro en ensayo de migración y de $9.47 \times 10^{-8} \mathrm{~cm}^{2} / \mathrm{s}$ para la molécula de oxígeno. Así pues, los coeficientes de difusión efectivos obtenidos para los iones cloruro mediante ensayos de difusión natural y migración son muy similares mientras que el de difusión de oxígeno es claramente superior.

Estos resultados permiten deducir que la migración de los iones cloruro se produce de forma similar y a través de poros del mismo tamaño que por los que se lleva a cabo la difusión natural; es decir, la
Cl difusion natural ( $\mathrm{mmol})$ carga acumulada $(\mathrm{Q})$

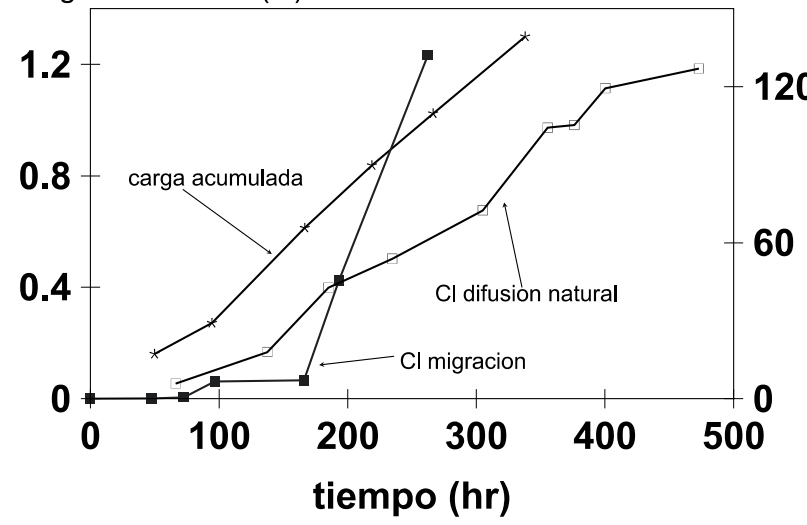

Figura 1: Evolución de la cantidad de ión cloruro en el compartimento anódico para el ensayo de migración, en el compartimento destino en los ensayos de difusión natural, y carga total acumulada correspondiente al consumo catódico de oxígeno.

aceleración que el campo eléctrico aplicado (hasta $10.5 \mathrm{~V} / \mathrm{cm}$ ) imprime a los iones cloruro, no altera las interacciones electrostáticas de los mismos con las paredes de los poros. Por consiguiente, en lo que a este aspecto se refiere, los coefientes de difusión de los iones cloruro a través de pastas de cemento no se verían afectados por su determinación mediante ensayos de migración.

En la figura 2-a se presentan los cloruros que quedan libres en la disolución de los poros del hormigón en función de los cloruros totales en la muestra después de someterlos a migración en estado no estacionario (ambos en porcentaje respecto a total de muestra). Se aprecia que inicialmente, todos los cloruros quedan libres en disolución, no existiendo combinación de los mismos hasta que el contenido en cloruros totales alcanza un valor en torno al $0.14 \%$ sobre peso de muestra, en que comienza la retención por parte de las fases sólidas. Por diferencia entre los cloruros totales y los libres se obtienen los combinados que, en función de la relación entre las concentraciones de cloruros en la disolución de los poros y en una disolución de $\mathrm{NaCl}$ saturada a la temperatura de la adsorción, se presentan en la figura 2-b.

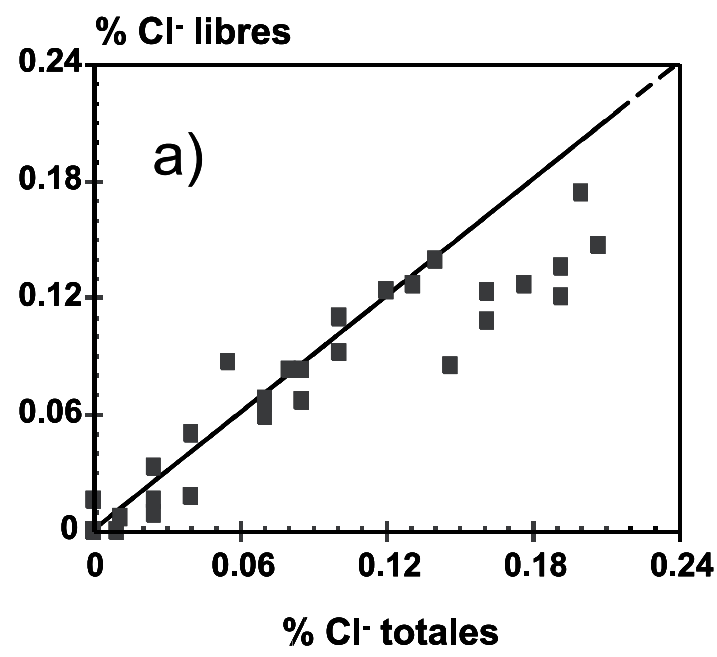

\section{$\% \mathrm{Cl}^{-}$combinados}

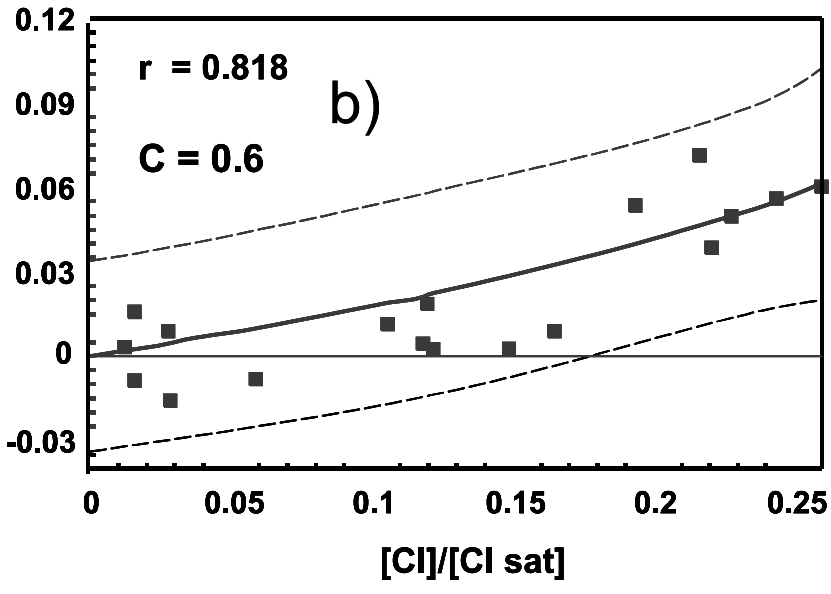

Figura 2 (a-b): a) Cloruros libres en función de los cloruros totales después de migración en estado no estacionario (ambos en porcentaje respecto a total de muestra). b) Ajuste de los datos experimentales a la isoterma BET. 
Para cuantificar la capacidad de retención de cloruros por parte de la matriz se han ajustado los datos según la isoterma de adsorción BET general [19] adaptándola, para la adsorción a partir de líquidos, según el procedimiento descrito en [20] (ver figura 2-b). Aunque el ajuste no es muy bueno (coeficiente de correlación de 0.818 ) se aprecia que todos los datos están dentro del rango correspondiente al $95 \%$ de confianza, y los residuos están en el rango de \pm 0.03 unidades lo que, para un material tan heterogéneo como el hormigón, y teniendo en cuenta los errores experimentales implícitos en las determinaciones de cloruros, es una aproximación suficientemente buena.

El valor de la constante característica de la isoterma obtenido, $\mathrm{C}=0.6<2$, informa de que se trata de una isoterma tipo III o tipo $\mathrm{V}$, característica de interacción débil y no favorable del adsorbato con el sustrato. Por el contrario, los valores de $\mathrm{C}$ encontrados para ensayos de difusión natural y ajustados a isoterma BET fueron por encima de 50 en todos los casos, que corresponden a isotermas tipo II o tipo IV, características de interacciones favorables entre adsorbato y adsorbente [20].

Con objeto de clarificar más lo anterior y hacer más hincapié en las diferencias entre difusión natural y migración, en lo que a retención de cloruros por parte de la matriz se refiere, se ha representado la curva de cloruros combinados (\% vs peso cemento) en función de la concentración de cloruro en la fase acuosa de los poros obtenidas a partir de la isoterma hallada en el presente trabajo de investigación junto a la isoterma de Langmuir propuesta por Sergi y col [16] para ensayos de penetración de cloruros en pasta de cemento (también OPC) por difusión natural (ver figura 3).

La representación conjunta de estas dos curvas, lleva, por un lado, a que en determinadas condiciones, mediante migración se llega a obtener el mismo volumen de cloruros combinados que a partir de difusión natural. El punto de cruce se produce (para los ensayos llevados a cabo en el presente trabajo) a una concentración de cloruro libre del $0.28 \%$ vs peso de muestra, es decir, para aproximadamente un $0.48 \%$ de cloruros totales en la muestra. Este valor, aunque no se puede tomar como absoluto (ya que se trata de ensayos realizados sobre distinto tipo de material, con distintas partidas de cemento y en distintas condiciones) sí representa un punto de referencia. Por otra parte, de la observación de esta figura se deduce que, lógicamente tiene que haber un punto de inflexión en la isoterma de migración, con lo que el tipo final encontrado sería el V.

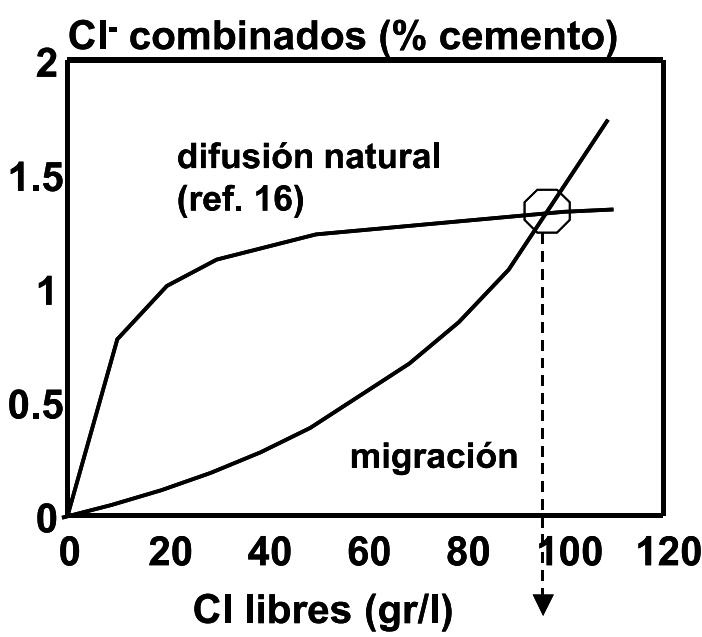

\section{$0.5 \%$ peso muestra}

Figura 3: Comparación entre la capacidad de combinación de cloruros si penetran por difusión natural y migración
Esta diferencia en el proceso de combinación de los cloruros entre migración y difusión natural se atribuye a la distinta velocidad relativa entre las etapas de transporte y combinación: En difusión natural, el proceso de combinación y/o retención de cloruros es rápido si se compara con la difusión de los iones por lo que la difusión de los iones es la etapa controlante del proceso global, lo que deja de cumplirse al acelerar el movimiento de los iones mediante la aplicación de un campo eléctrico. Dado que el coeficiente de difusión aparente depende del grado de combinación, lo anteriormente expuesto hace ver que el acelerar el transporte de los cloruros por migración sí influirá en los coeficientes de difusión obtenidos, debiéndose tener en cuenta a la hora de diseñar y comparar los coeficientes obtenidos a partir de ensayos de difusión natural y de migración.

\section{CONCLUSIONES}

Las conclusiones relativas a las alteraciones que causa la aplicación de corriente eléctrica en las interacciones matriz/cloruros y que se extraen a partir de los resultados obtenidos son las siguientes :

1. La aceleración que sufren los iones cloruro por la acción del campo eléctrico aplicado (hasta $10.5 \mathrm{~V} / \mathrm{cm}$ ) no es capaz de vencer las interacciones electrostáticas de los mismos con las paredes de los poros. Por tanto, cuando el transporte de los iones se produce por migración, ésta tiene lugar a través de poros del mismo tamaño que por los que se lleva a cabo la difusión natural.

2. Cuando los cloruros penetran en el hormigón por migración se produce una interacción más débil del adsorbato con el sustrato (isoterma BET tipo V), al contrario de lo que ocurre en difusión natural en que los valores encontrados se ajustan a isotermas características de interacciones favorables. A pesar de ello, para concentraciones suficientemente altas de ión cloruro (entre el $0.25-0.30 \%$ de cloruros libres sobre peso de muestra), la combinación que tiene lugar durante un ensayo de migración reproduce en gran medida la que se produce por difusión natural.

\section{AGRADECIMIENTOS}

Los autores quieren agradecer su ayuda al Dr. C. L. Page, a Gavin Chadbourn y al Departamento de Ingeniería Civil de la Universidad de Aston, Birmingham, donde se llevó a cabo el trabajo experimental correspondiente a la difusión de oxígeno que se incluye en este artículo.

\section{BIBLIOGRAFÍA}

1. C. Andrade "Calculation of chloride diffusion coefficients in concrete from ionic migration measurements, Cement and Concrete Research, 23, $\mathrm{N}^{\mathrm{o}} 3$, (1993) 724-742

2. M. Castellote, C. Alonso, C. Andrade "Chloride binding isotherms in concrete submitted to non-steady-state migration experiments" Cement and Concrete Research. Vol 29, pp 416.421 (1999).

3. M. Castellote, C. Alonso, C. Andrade G. Chadbourn and C.L. Page. "Oxygen and chloride diffusion in cement pastes as a validation of chloride diffusion coefficients obtained by steady-state migration tests" Cement and Concrete Research, 31 (2001) 621-625.

4. H. Londiche, F. Lancelot, Les phénomènes électrocinétiques et leurs aplications aux écoulements dans les milieux poreux, Annales des Mines, MaiJuin, (1984), 103-108.

5. E. Nägele, The zeta-potential of cement - part III: The non-equilibrium double layer on cement, Cement and Concrete Research, 17, № 4, (1987) 573-580.

6. S. Chatterji, M. Kawamura, Electrical double layer, ion transport and reactions in hardened cement paste. Cement and Concrete Research, 22, $\mathrm{N}^{\circ} 5$, (1992), 774-782. 
7. C.L. Page, N.R. Short, A. Tarras, Diffusion of chloride ions in hardened cement pastes, Cement and Concrete Research, 11, N 3, (1981), 395-406.

8. S. Goto, D.M. Roy, Diffusion of ions through hardened cement pastes, Cement and Concrete Research, 11, (1981) 751-757.

9. S.W. Yu, C.L. Page, Diffusion in cementitious materials I: Comparative study of chloride and oxygen diffusion in hydrated cement pastes. Cement and Concrete Research, 21, (1991) 581-588.

10. V. T. Ngala, C. L. Page, L.J. Parrott, S.W. Yu, Diffusion in cementitius materials II: Further investigations of chloride and oxigen diffusion in well cured OPC and OPC/30\% PFA pastes, Cement and Concrete Research, 25, $\mathrm{N}^{\circ} 4$, (1995) 819-826.

11. V.S. Ramachandran: "Possible states of chloride in the hydration of tricalcium silicate in the presence of calcium chloride". Materials and Structures, Vol 4, No 19, (1971) 3-12.

12. E.M. Theissing; T. Mebius-Van de Laar; G. De Wind: "The combining of sodium chloride and calcium chloride by the hardened portland cement compounds $\mathrm{C}_{3} \mathrm{~S}, \mathrm{C}_{2} \mathrm{~S}, \mathrm{C}_{3} \mathrm{~A}$ and $\mathrm{C}_{4} \mathrm{AF}^{\prime \prime}$. 7th International Congress on the Chemistry of Cement, Vol IV, Paris (1980) pp 823-828.

13. C.L. Page; O. Vennesland: "Pore solution composition and chloride binding capacity of silica fume pastes". Materials and Structures / Matériaux et Constructions, Vol 16, No 91, (1983) 19-25.
14. K. Byfors: "Chloride binding in cement paste". Nordic Concrete Research, Vol 5, (1986) 27-38.

15. F.P. Glasser; K Luke; M.J. Angus: "Modification of cement pore fluid compositions by pozzolanic additives". Cement and Concrete Research, Vol 18, (1988) 165-178.

16. G. Sergi; S.W. Yu; C.L. Page: "Diffusion of chloride and hydroxil ions in cementitius materials exposed to a saline environment". Magazine of Concrete Research, 44, No 158, (1992) 63-69.

17. C.L. Page; O. N. R. Short, A. Tarras: "Diffusion of chloride ions in hardened cement pastes". Cement and Concrete Research, Vol 11, No 3, pp 395-406, (1981).

18. M. Castellote, C. Andrade and C. Alonso. "Changes in the concrete pore size distribution due to electrochemical chloride migration trials" ACI Materials Journal, no 96-M39, Mayo/Junio, 1999.

19. S. Brunauer, L.S. Dewing, W.E. Dewing, E.J. Teller, : "On a theory of the Van der Waals adsorption of gases". J. Amer. Chem. Soc., 62, pp 1723-1732, (1940).

20. L. Tang; L.O. Nilsson: "Chloride binding isotherms - An approach by applying the modified BET equation". Proceedings of the RILEM International Workshop on Chloride Penetration into Concrete, Oct. 15-18, St. Rémyle-Chevreuse, (1995).

Recibido: 1.2 .03

Aceptado: 30.11 .03 